\title{
MONITORAMENTO E INTENSIDADE DAS SECAS E CHUVAS NA CIDADE DE CAMPINA GRANDE/PB
}

MACEDO, Maria José Herculano - mariejhm@hotmail.com Universidade Federal de Campina Grande / Paraíba

GUEDES, Roni Valter de Souza - roniguedes84@yahoo.com.br Universidade Federal de Campina Grande / Paraíba

SOUSA, Francisco de Assis Salviano - fassis@dca.ufcg.edu.br Universidade Federal de Campina Grande / Paraíba

\begin{abstract}
RESUMO
A cidade de Campina Grande no estado da Paraíba se encontra numa zona de transição entre os climas do Litoral e do Sertão e por isso detém uma alta irregularidade pluviométrica. Nesse sentido, o objetivo desse trabalho foi realizar uma análise da variabilidade temporal das chuvas e secas em diferentes escalas temporais na cidade de Campina Grande-PB. Para tanto foi utilizada uma série histórica de totais mensais precipitados entre os anos de 1962 e 2009 observados na estação da Embrapa do município em estudo, para o cálculo do índice SPI nas escalas temporais de 01, 03, 06, 12 e 24 meses. Os resultados revelaram que os SPIs analisados não detectaram, segundo a intensidade média, categorias de secas e chuvas extremas na cidade de Campina Grande. A categoria mais freqüente entre os SPIs foi a de chuva normal. Em termos quantitativos as chuvas anuais na cidade de Campina Grande sempre ocorrem em torno do valor esperado. Essa esperança matemática ocorre com maior freqüência pelo lado negativo da escala em torno do valor médio. Esse é o motivo da característica semiárida da Cidade. Os anos que apresentaram maiores e menores índices de pluviometria foram detectados pelo SPI-12 meses e guardam uma boa relação com os eventos La Niña e El Niño, respectivamente, revelando uma boa representatividade em relação às condições reais da pluviometria local. As secas dos períodos de 1968 a 1971, de 1988 a 1989 e de 1997 a 2000 foram identificadas por todos os SPIs estudados. Da mesma forma, os períodos de 1963 a 1969 e de 2004 a 2005 foram detectados como chuvosos por todos os SPIs.
\end{abstract}

Palavras-Chave: Precipitação pluvial, SPI, El Niño, La Niña.

MONITORING AND INTENSITY OF DROUGHTS AND RAINS IN THE CITY OF CAMPINA GRANDE / $P B$

\section{ABSTRACT}

The city of Campina Grande in Paraíba state is in a transition zone between the climates of the Coast and Hinterland and therefore has a high rainfall irregularity. In this sense, the objective of this study was an analysis of the temporal variability of rainfall and drought on different time scales in the city of Campina Grande-PB. Using it for a series of monthly precipitation totals between 1962 and 2009 observed at the station of Embrapa in the city study, for calculating the index in SPI time scales of $01,03,06,12$ and 24 months. The results revealed that the analysis did not detect SPIs, according to the average intensity, categories of drought and extreme rainfall in the city of Campina Grande. The most frequent category among the SPIs was for normal rainfall. The volume of annual rainfall in the city of Campina Grande always occur around the expected value. This mathematical expectation occurs most often on the negative side of the scale around the mean value. This is why the characteristic of semiarid City. The years had higher and lower rates of rainfall were detected by the SPI-12 months and keep a good relationship with the events El Niño and La Niña, respectively, showing a good representation in relation to the actual conditions of local rainfall. Drought periods from 1968 to 1971 , from 1988 to 1989 and from 1997 to 2000 were identified by all SPIs studied. Equally, the periods from 1963 to 1969 and from 2004 to 2005 were detected as rainy by all SPIs.

Keywords: rainfall, SPI, El Niño, La Niña.

\section{INTRODUÇÃO}

O Estado da Paraíba possui cerca de $80 \%$ da sua área inserida no semiárido nordestino e apresenta alta irregularidade pluviométrica (Azevedo \& Silva, 2004). A delimitação do semiárido tem como critérios o 
índice pluviométrico, o índice de aridez e o risco de seca (Brasil, 2005). O Agreste paraibano é tido como uma área de transição entre a zona da mata e a zona das caatingas, com trechos quase tão úmidos como no litoral e outros secos como no sertão (Pereira et al., 2002), essa semelhança com o clima do Litoral e do Sertão revelam o quanto as chuvas nessa sub-região apresentam variabilidade espaço-temporal e dessa forma dificulta ainda mais a previsibilidade e monitoramento dos eventos de secas/chuvas no âmbito do semiárido paraibano.

Entre os anos de 1979 a 1983 e 1998 e 1999, o sistema de abastecimento de água da cidade de Campina Grande, localizada no Agreste paraibano, quase entrou em colapso, devido a eventos de seca na região. Seria uma situação embaraçosa para a segunda maior cidade do Estado, considerada um grande pólo tecnológico e industrial e responsável por cerca de 13,6\% do PIB do Estado (IBGE, 2008). Além disso, polariza uma região metropolitana com aproximadamente 800 mil habitantes. Apesar de se encontrar numa zona de transição, o município está incluído na área geográfica de abrangência do semiárido brasileiro, definida pelo Ministério da Integração Nacional de acordo com (Brasil, 2005). O clima de Campina Grande é tropical semiárido e a altitude é de 552 metros acima do nível médio do mar e $594 \mathrm{~km}^{2}$ de área e apresenta em sua área o Bioma Caatinga (IBGE, 2011). Apresenta temperaturas mais amenas durante todo o ano, variando de 16 a $32{ }^{\circ} \mathrm{C}$. A umidade relativa do ar média é cerca de $80 \%$.

O Índice Padronizado de Precipitação (Standardized Precipitation Index SPI) foi desenvolvido por Mckee et al. (1993) objetivando o monitoramento das secas/chuvas em diferentes escalas de tempo, utilizando para isto apenas dados mensais de precipitação, mostrando com isso vantagem em relação aos demais. Este índice teve destaque no Workshop Inter-Regional sobre Índices e Sistemas de Alerta Precoce de Seca, realizada na Universidade de Nebraska-Lincoln, E.U.A., através de um acordo de consenso de que o Índice Padronizado de Precipitação (SPI) deveria ser usado para caracterização das secas meteorológicas por todos os Serviços Nacionais Meteorológicos e Hidrológicos em todo o mundo (Press Release No. 872).

O SPI vem sendo usado em vários estudos científicos devido a sua capacidade de quantificar o déficit ou excesso pluviométrico em diversas escalas temporais e de permitir comparações entre regiões com diferentes características climáticas (MCKEE et al., 1993; TSAKIRIS \& VANGELIS, 2004; TONKAZ, 2006; BLAIN \& BRUNINI, 2007; MACEDO et al., 2010). Tsakiris \& Vangelis (2004) apresentaram um método de estimativa do índice SPI (03, 06 e 12 meses) para uma área geográfica, na parte Este da Ilha de Creta, e sua utilização para caracterização das secas nessa área, observando que o procedimento proposto pode caracterizar uma área de dimensões de mesoescala. Tonkaz (2006) estudou o comportamento das secas na parte sudeste da Turquia, usando o SPI. O autor identificou que os eventos de secas atuantes nos anos 1999-2000 influenciaram o decréscimo das atividades agrícolas em comparação com a década anterior. Blain \& Brunini (2007) realizaram análises comparativas entre os índices SPI, PDSI e PDSI adaptado, eles concluíram que o SPI é mais versátil para o cálculo do déficit hídrico em diversas escalas de tempo do que os outros métodos. Macedo et al. (2010) utilizaram SPIs (12 e 24 
meses) para monitorar as secas severas e extremas nas sub-regiões pluviométricas homogêneas do Estado da Paraíba.

A distribuição da precipitação pluvial está associada à interação entre a atmosfera, os oceanos e a fisiografia, como evidenciado em anos de fenômenos El Niño e La Niña e nas alterações do Dipolo do Atlântico. Além disso, é interessante salientar que de alguma forma esses fenômenos tem uma influência pontual (Cerqueira, 2010; Neto et al. 2007).

Assim, o objetivo deste trabalho é realizar uma análise da variabilidade temporal das chuvas e secas em diferentes escalas temporais na cidade de Campina Grande-PB.

\section{MATERIAL E MÉTODOS}

Foram usados os totais mensais precipitados observados na estação da Embrapa, localizada no município de Campina Grande-PB (7014'S, 35054'W e $552 \mathrm{~m}$ ), região Agreste paraibana (Figura 1). Esses dados de chuvas se encontram disponíveis na Unidade Acadêmica de Ciências Atmosféricas (UACA) da Universidade Federal de Campina Grande (UFCG). A série de dados apresenta período de registro entre os anos de 1962 e 2009.

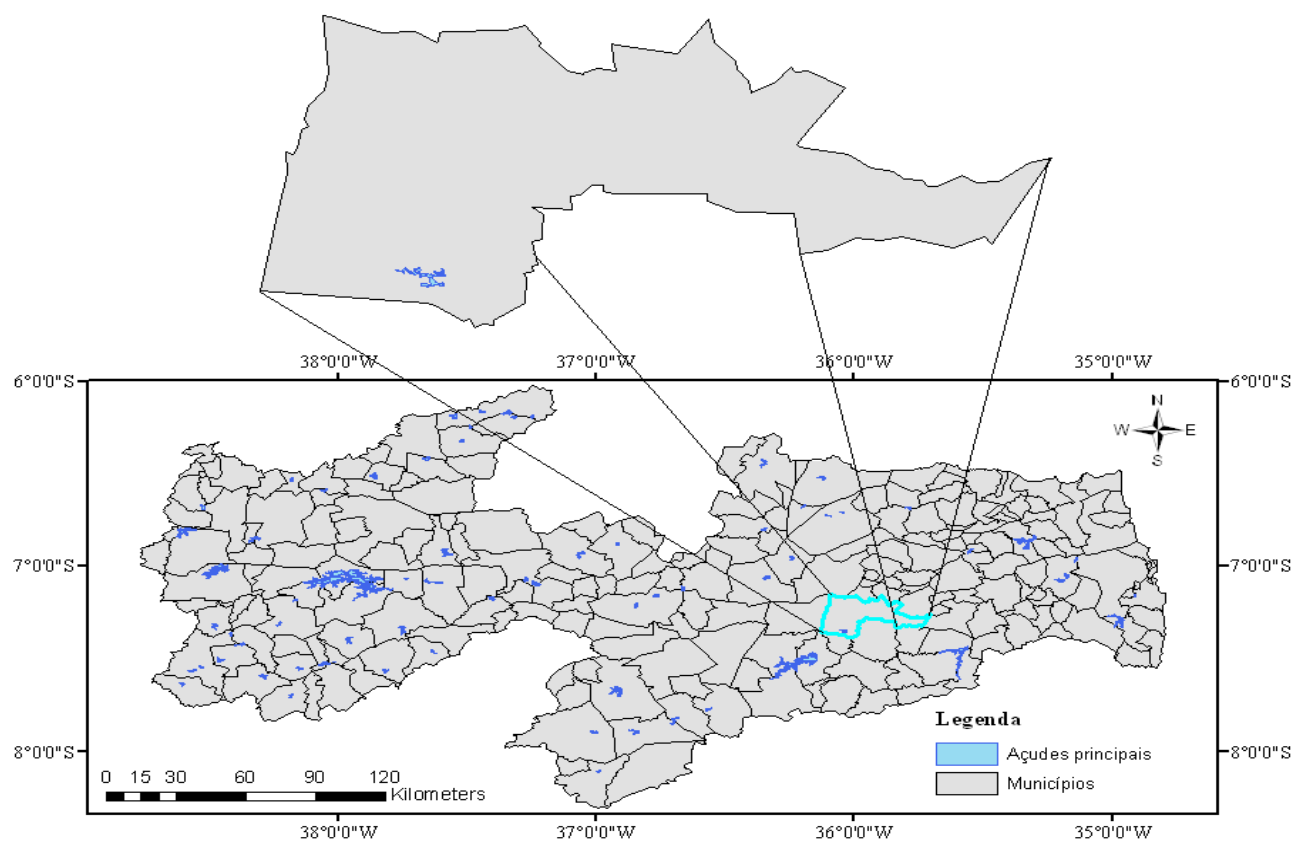

Figura 1. Localização geográfica da cidade de Campina Grande - PB.

\section{Índice Padronizado de Precipitação (SPI)}

O cálculo do SPI é determinado a partir das funções de densidade de probabilidade que descrevem as séries históricas de precipitação nas diferentes escalas de tempo. Assim, foram consideradas as distribuições normal e Gama, para fins de ajustar uma dada distribuição de frequência ao total precipitado em dado posto pluviométrico, assim, a função distribuição de probabilidade Gama é dada por: 


$$
g(x)=\frac{1}{B^{a} \Gamma(a)} x^{a-1} e^{-x / \mathrm{b}}
$$

em que a $>0$ é o parâmetro de forma; b $>0$ é o parâmetro de escala e $x$ $>0$ é a quantidade de chuva precipitada. A função Gama é dada por:

$$
\Gamma(a)=\int_{0}^{\infty} y^{a-1} e^{-y} d y
$$

Os parâmetros a e b da função densidade de probabilidade Gama são estimados para cada estação e escala de tempo de interesse. Para estimativa dos parâmetros a e b, utilizam-se as soluções de Máxima Verossimilhança, sendo:

$$
\hat{a}=\frac{1}{4 A}\left(1+\sqrt{1+\frac{4 A}{3}}\right) \quad \text { em que } A=\ln (\bar{x})-\frac{\sum_{i}^{n} x_{i}}{n}, \quad \text { e, } \hat{b}=\frac{\bar{x}}{\hat{a}}
$$

n é o número de observações da amostra e $\bar{x}$ é o valor médio dos dados de chuva.

Os parâmetros resultantes serão utilizados para obtenção da probabilidade de chuva para um determinado mês e para o período de tempo desejado para o posto em estudo. Logo, a função acumulada de probabilidade Gama é:

$$
G(x)=\frac{1}{\Gamma(\hat{a})} \int_{0}^{x} t^{\hat{a}-1} e^{-t} d t
$$

A função Gama $\Gamma(a)$ não é definida para $\mathrm{x}=0$, mas como a amostra dos dados de precipitação pode conter zeros, a probabilidade acumulada é dada da seguinte forma:

$$
H(x)=q+(1-q) G(x)
$$

em que $q$ é a probabilidade de ocorrer um zero. Se $m$ for o número de zeros numa série de precipitação, então $q=m / n$. Segundo Abramowitz e Stegun (1965), a relação entre as distribuições de probabilidade Gama e Normal é dada por:

$$
\begin{aligned}
& Z=S P I=-\left(t-\frac{c_{0}+c_{1} t+c_{2} t^{2}}{1+d_{1} t+d_{2} t^{2}+d_{3} t^{3}}\right) \quad \text { para } 0<\mathrm{H}(\mathrm{x}) \leq 0,5 \\
& Z=S P I=+\left(t-\frac{c_{0}+c_{1} t+c_{2} t^{2}}{1+d_{1} t+d_{2} t^{2}+d_{3} t^{3}}\right) \quad \text { para } 0,5<\mathrm{H}(\mathrm{x}) \leq 1,0
\end{aligned}
$$


sendo, $c_{0}=2,515 ; c_{1}=0,803 ; c_{2}=0,010 ; d_{1}=1,433 ; d_{2}=0,189 ; d_{3}=$ 0,001 ;

$$
\begin{array}{ll}
\text { em que } t=\sqrt{\ln \left(\frac{1}{(H(x))^{2}}\right)} & \text { para } 0<\mathrm{H}(\mathrm{x}) \leq 0,5 \\
\text { e } \quad t=\sqrt{\ln \left(\frac{1}{(1-H(x))^{2}}\right)} & \text { para } 0,5<\mathrm{H}(\mathrm{x}) \leq 1,0
\end{array}
$$

O cálculo do SPI inicia com o ajuste da função densidade de probabilidade Gama às séries de totais mensais precipitados. Em seguida, a probabilidade acumulada de ocorrência de cada total mensal é estimada. A função Normal inversa Gaussiana é aplicada a essa probabilidade resultando no valor SPI. O SPI pode ser calculado para diferentes escalas de tempo, significando o período durante o qual se acumula o valor da precipitação. Assim, o SPI-01 corresponde à precipitação mensal, o SPI-03 corresponde à precipitação acumulada em períodos de 3 meses etc. $O$ evento "chuva" se inicia quando o SPI torna-se positivo e atinge o valor +1 e finda quando este volta a apresentar valores negativos. Em contrapartida, o evento seca se inicia quando o SPI torna-se negativo e atinge o valor -1 e finda quando este volta a apresentar valores positivos. Dentro de sua escala, os valores maiores ou iguais a +2 indicam "chuva extrema" e os maiores ou iguais a -2 indicam "seca extrema" (Tabela 1).

Tabela 1. Classificação dos períodos secos e chuvosos do SPI.

\section{Classificação do SPI}

\begin{tabular}{cc}
\hline$\geq 2.00$ & Chuva extrema \\
1.99 a 1.50 & Chuva severa \\
1.49 a 1.00 & Chuva moderada \\
0.99 a 0.50 & Chuva fraca \\
0.49 a -0.49 & Normal \\
-0.50 a -0.99 & Seca fraca \\
-1.00 a -1.49 & Seca moderada \\
-1.50 a -1.99 & Seca severa \\
$\leq-2.00$ & Seca extrema \\
\hline 1993), Hughes \& Saunders (2002) e Domingos (2006).
\end{tabular}

Fonte: Mckee (1993), Hughes \& Saunders (2002) e Domingos (2006).

Os climas úmidos e secos podem ser representados, pois o SPI é normalizado. Sendo assim, o SPI pode monitorar tanto os períodos úmidos quanto os secos (Tsakiris \& Vangelis, 2004; Macedo et al., 2010).

\section{RESULTADOS E DISCUSSÃO}

A precipitação média anual da cidade de Campina Grande é igual a 804,9 $\mathrm{mm}$, com valores mensais concentrados (superiores a $100 \mathrm{~mm}$ ) durante os meses de março a julho, enquanto o trimestre de menor pluviometria varia de outubro a dezembro (Figura $2 \mathrm{a}$ ). $\mathrm{Na}$ série histórica de pluviometria os anos que apresentaram maiores valores (Figura $2 b$ ) foram 
1964, 1985, 1986, 2000, 2004 e 2009. Nesses anos, com exceção de 2000, houve a atuação do fenômeno La Niña. Em 2000 o ano é considerado normal e em 2009 ocorreu o evento El Nino, categorizado como fraco. Na Figura 2c pode ser visto que o maior valor pluviométrico ocorreu no ano 2000 . Já os anos de menor pluviometria são 1993 e 1998, com totais anuais inferiores a $400 \mathrm{~mm}$. Esses anos foram influenciados pelo fenômeno El Niño. Esses anos secos foram detectados pelo SPI-12 meses (Figura 2c). Comparando-se as Figuras 2 b e $2 c$ nota-se uma boa representatividade do SPI em relação às condições reais da pluviometria local.

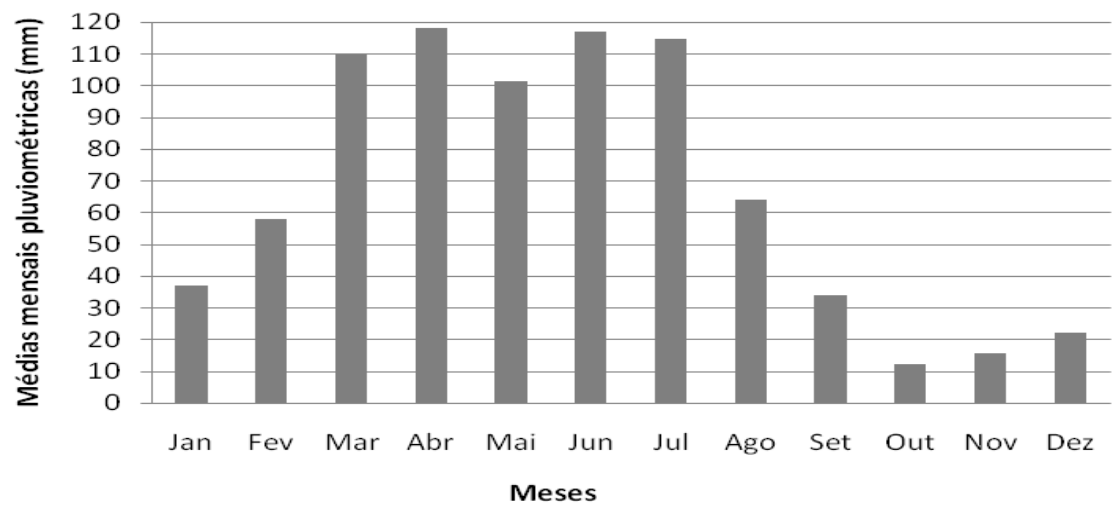

a.

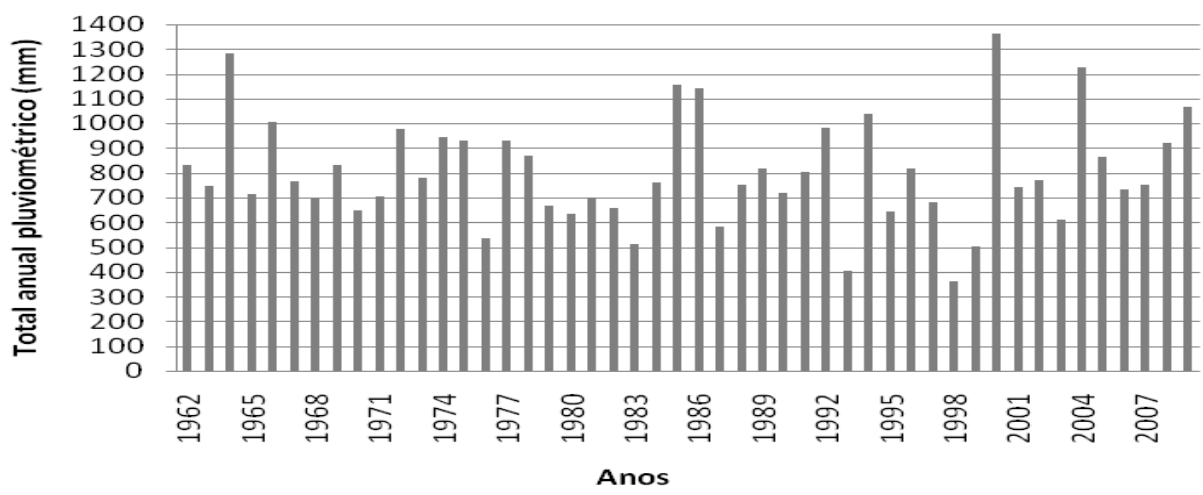

b.

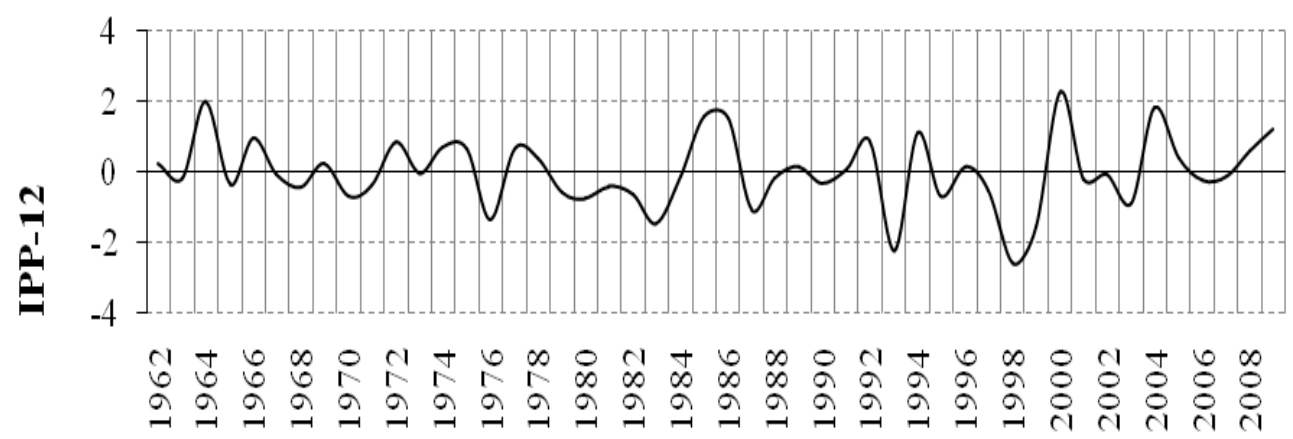

Anos

c.

Figura 2 - Características pluviométricas da cidade de Campina Grande, médias 
mensais (a), totais anuais precipitados (b) e ÍPP - 12 meses (c).

\section{III.1 - Análises das secas}

O SPI-01 mês revela índices semelhantes às médias mensais pluviométricas, portanto essa escala temporal não é tão importante quanto às escalas temporais maiores. Então, foi dado preferência as escalas superiores a três meses.

O SPI detecta ocorrência de seca como chuva acumulada abaixo da média e classifica sua categoria de acordo com a intensidade média do período de duração do evento. O pico de intensidade equivale ao valor máximo da seca em um determinado mês, durante sua ocorrência. A Tabela 2 exibe os resultados do SPI-03. Pode ser visto que no trimestre a chuva acumulada gerou vários tipos de secas com durações distintas. Esses resultados já eram esperados haja vista o período de estiagem na cidade. Nessa escala temporal é interessante destacar apenas as secas com durações e intensidades maiores.

Pode ser visto na Tabela 2 que a seca com maior duração foi de 26 meses, no período de 1982 a 1984, na categoria fraca. Em termos de magnitude, a precipitação se manteve abaixo da média durante esses anos. Foram detectadas, também, algumas secas moderadas, mais significativas em termos de aridez, principalmente se a duração for longa. A mais forte ocorreu entre 1992 e 1994, durante 17 meses e com pico de -3,09. Os piores períodos no trimestre foram detectados por duas secas severas, a primeira de 1968 a 1969, durante 11 meses, e a segunda em 1998 durante 9 meses. Nesses anos houve a influência do fenômeno El Niño. Em 1998 há destaque para o El Niño mais intenso da história (Figura 2) (El Niño e La Niña, 2010).

Tabela 2. Períodos, duração, intensidade e caracterização de secas obtidas pelo SPI-03.

\begin{tabular}{|c|c|c|c|c|c|}
\hline \multicolumn{2}{|c|}{ Anos } & \multirow{2}{*}{$\begin{array}{l}\text { Duração } \\
\text { (meses) }\end{array}$} & \multirow{2}{*}{$\begin{array}{c}\text { Intensidade } \\
\text { do Pico }\end{array}$} & \multirow{2}{*}{$\begin{array}{c}\text { Intensidade } \\
\text { média }\end{array}$} & \multirow{2}{*}{$\begin{array}{c}\text { Categoria de } \\
\text { seca }\end{array}$} \\
\hline Inicial & Final & & & & \\
\hline 1964 & 1965 & 5 & -1.16 & -0.69 & Fraca \\
\hline $1968 *$ & 1969* & $11^{*}$ & $-3.00 *$ & $-1.54 *$ & Severa* \\
\hline 1969 & 1970 & 4 & -1.99 & -1.18 & Moderada \\
\hline 1970 & 1971 & 7 & -2.03 & -1.41 & Moderada \\
\hline 1976 & 1976 & 8 & -3.09 & -1.25 & Moderada \\
\hline 1977 & 1978 & 4 & -1.53 & -0.60 & Fraca \\
\hline 1978 & 1979 & 6 & -1.69 & -0.79 & Fraca \\
\hline 1980 & 1980 & 6 & -1.93 & -1.06 & Moderada \\
\hline 1981 & 1981 & 5 & -2.27 & -1.96 & Severa \\
\hline 1982* & 1984* & $26 *$ & $-1.81 *$ & $-0.62 *$ & Fraca* \\
\hline 1987 & 1988 & 14 & -1.48 & -0.75 & Fraca \\
\hline 1989 & 1989 & 3 & -1.93 & -1.04 & Moderada \\
\hline 1990 & 1990 & 3 & -1.25 & -1.10 & Moderada \\
\hline 1990 & 1991 & 4 & -1.49 & -0.86 & Fraca \\
\hline 1992* & 1994* & $17^{*}$ & $-3.09 *$ & $-1.18 *$ & Moderada* \\
\hline 1995 & 1995 & 4 & -1.30 & -0.74 & Fraca \\
\hline
\end{tabular}




\begin{tabular}{cccccc}
1995 & 1996 & 7 & -2.21 & -0.96 & Fraca \\
1997 & 1997 & 7 & -1.55 & -0.82 & Fraca \\
$199 *^{*}$ & $1998^{*}$ & $9 *$ & $-3.22^{*}$ & $-1.51^{*}$ & Severa* \\
1998 & 1999 & 13 & -2.66 & -1.00 & Moderada \\
2002 & 2002 & 7 & -1.19 & -0.42 & Fraca \\
2003 & 2003 & 9 & -1.27 & -0.64 & Fraca \\
2004 & 2005 & 6 & -1.37 & -0.56 & Fraca \\
2007 & 2008 & 3 & -1.29 & -0.57 & Fraca \\
2008 & 2009 & 3 & -1.07 & -0.53 & Fraca \\
\hline
\end{tabular}

* datas, valores e categoria referenciadas no texto deste artigo.

Existe uma boa relação entre os eventos de seca e anos de El Nino. Ao se comparar os índices da Tabela 2 com os da Figura 3, nota-se que todas as secas ocorreram nos anos com desvios positivos da TSM.

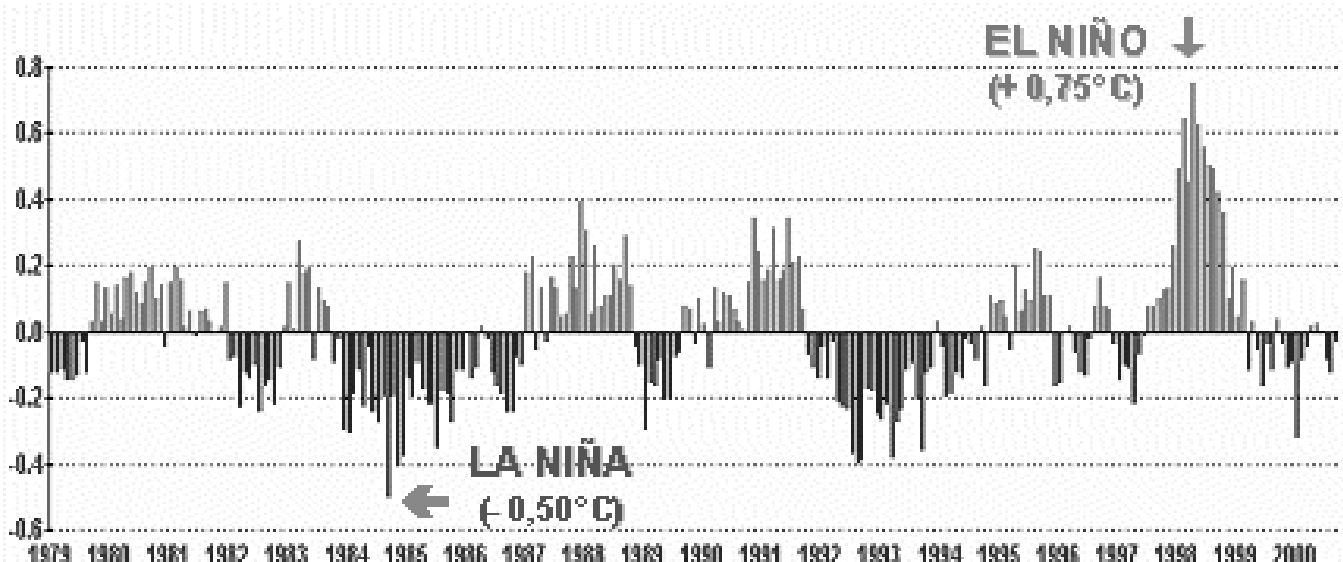

Figura 3 - Desvios da TSM obtidos por satélite.

Fonte: www.ghcc.mspc.nasa.gov

Na Tabela 3 pode-se notar que algumas secas detectadas pelo SPI-03 não permanecem no SPI-06, isso significa que ocorreu precipitação no período maior, seis meses, desse modo, as secas que persistiram nas escalas maiores provocaram maiores impactos na disponibilidade hídrica local. Também, é interessante notar que algumas categorias de secas mudaram. Por exemplo, as duas secas severas do SPI-03, Tabela 2, período de 1968 a 1969 transformou-se em uma moderada no SPI-06, Tabela 3, e a segunda de 1998 transformou-se também em moderada no ano de 1999, com 31 meses de duração, apresentando pico igual a -3.19. Ainda pode ser visto na Tabela 3 que as secas fracas (1882 a 1984) e moderada (1992 a 1994), destacadas na Tabela 2, reaparecem na Tabela 3 . Os inícios dessas secas coincidem com a atuação do fenômeno El Nino (Ocorrência de El Niño, 2010). Na Tabela 3 destaca-se a quantidade de secas fracas identificadas pelo SPI - 06.

Tabela 3. Períodos, duração, intensidade e caracterização de secas obtidas pelo SPI-06. 


\begin{tabular}{|c|c|c|c|c|c|}
\hline \multicolumn{2}{|c|}{ Anos } & \multirow{2}{*}{$\begin{array}{l}\text { Duração } \\
\text { (meses) }\end{array}$} & \multirow{2}{*}{$\begin{array}{c}\text { Intensidade } \\
\text { do Pico }\end{array}$} & \multirow{2}{*}{$\begin{array}{c}\text { Intensidade } \\
\text { média }\end{array}$} & \multirow{2}{*}{$\begin{array}{c}\text { Categoria de } \\
\text { seca }\end{array}$} \\
\hline Inicial & Final & & & & \\
\hline 1965 & 1965 & 7 & -1.41 & -0.53 & Fraca \\
\hline 1968* & 1969* & $12 *$ & $-2.77^{*}$ & $-1.41^{*}$ & Moderada* \\
\hline 1970 & 1970 & 7 & -1.37 & -0.72 & Fraca \\
\hline 1970 & 1971 & 12 & -2.91 & -0.93 & Fraca \\
\hline 1976 & 1976 & 9 & -2.08 & -1.33 & Moderada \\
\hline 1979 & 1979 & 7 & -1.49 & -0.94 & Fraca \\
\hline 1980 & 1981 & 10 & -1.53 & -0.84 & Fraca \\
\hline 1981 & 1982 & 8 & -2.43 & -1.12 & Moderada \\
\hline $1982 *$ & 1984* & $27 *$ & $-1.46 *$ & $-0.77 *$ & Fraca* \\
\hline 1987 & 1988 & 17 & -1.9 & -0.74 & Fraca \\
\hline 1989 & 1989 & 7 & -1.51 & -0.51 & Fraca \\
\hline 1991 & 1991 & 2 & -1.12 & -0.77 & Fraca \\
\hline 1992* & 1994* & $20 *$ & $-3.15^{*}$ & $-1.23^{*}$ & Moderada* \\
\hline 1995 & 1996 & 5 & -2.22 & -0.94 & Fraca \\
\hline 1997* & 1999* & $31 *$ & $-3.19 *$ & $-1.34 *$ & Moderada* \\
\hline 2003 & 2003 & 9 & -1.06 & -0.75 & Fraca \\
\hline 2005 & 2005 & 5 & -1.24 & -0.56 & Fraca \\
\hline 2006 & 2006 & 4 & -1.00 & -0.57 & Fraca \\
\hline
\end{tabular}

* datas, valores e categoria referenciadas no texto deste artigo.

No SPI-12 o déficit hídrico atinge vários níveis da economia e impacta várias atividades humanas. Quando se alonga a duração o número de secas registradas é menor do que em outras escalas temporais. Na Tabela 4 pode visto que as secas detectadas pelos SPIs anteriores persistiram no SPI-12. Isso significa que elas merecem destaque em relação às demais. A seca de maior duração (57 meses) na categoria fraca ocorreu entre os anos de 1980 e 1985. Outras duas de maior impacto, na categoria severa, ocorreram no período de 1993 a 1994 e de 1997 a 2000, com durações de 16 e 36 meses, respectivamente. Resultados similares foram obtidos por Macedo et al. (2010). As categorias dessas duas secas foram elevadas de moderadas para severas, quando se compara o SPI-06 com o SPI-12. Ao se comparar os valores de precipitação média anual de toda a série com aqueles do período do SPI-12, verifica-se redução da disponibilidade hídrica durante o evento das secas. Essa redução de oferta de água obriga a tomada de decisão por parte do poder público no sentido de mitigar os problemas econômicos e sociais decorrentes dos eventos de secas. Destaca-se aqui a eficiência deste método em monitorar e detectar o início de secas em várias escalas temporais.

Tabela 4. Períodos, duração, intensidade e caracterização de secas obtidas pelo SPI-12.

\begin{tabular}{|c|c|c|c|c|c|}
\hline \multicolumn{2}{|c|}{ Anos } & \multirow{2}{*}{$\begin{array}{l}\text { Duração } \\
\text { (meses) }\end{array}$} & \multirow{2}{*}{$\begin{array}{c}\text { Intensidade } \\
\text { do Pico }\end{array}$} & \multirow{2}{*}{$\begin{array}{c}\text { Intensidade } \\
\text { média }\end{array}$} & \multirow{2}{*}{$\begin{array}{c}\text { Categoria de } \\
\text { seca }\end{array}$} \\
\hline Inicial & Final & & & & \\
\hline 1968* & 1969* & $14^{*}$ & $-2.38^{*}$ & $-0.81^{*}$ & Fraca* \\
\hline 1970 & 1972 & 21 & -1.31 & -0.7 & Fraca \\
\hline
\end{tabular}




\begin{tabular}{cccccc}
\hline 1976 & 1977 & 12 & -1.86 & -1.2 & Moderada \\
$1980 *$ & $1985^{*}$ & $57^{*}$ & $-1.73^{*}$ & $-0.78 *$ & Fraca* \\
1987 & 1989 & 30 & -1.48 & -0.55 & Fraca \\
$1993^{*}$ & $1994^{*}$ & $16^{*}$ & $-2.39 *$ & $-1.75^{*}$ & Severa* \\
$1997^{*}$ & $2000^{*}$ & $36^{*}$ & $-2.94 *$ & $-1.52^{*}$ & Severa* \\
2002 & 2003 & 17 & -1.1 & -0.54 & Fraca \\
\hline
\end{tabular}

* datas, valores e categoria referenciadas no texto deste artigo.

A Tabela 5 exibe os resultados do SPI-24, nela se destacam vários períodos críticos de secas. Dois deles na categoria fraca, um moderada e outro severa. Todas as secas apresentaram impactos significativos de curto a longo prazo. Destaca-se nessa escala, a seca moderada de 1981 a 1985, com duração de 48 meses. Também há ocorrência de uma seca fraca, com início em 1968 e final em 1972, com duração de 44 meses. Nesse período houve atuação do fenômeno El Niño com intensidade moderada (1968 a 1970) e forte (1982 e 1983). Apenas uma seca severa foi detectada pelo SPI-12, no período de 1997 a 2000, com duração de 38 meses. Os anos de 1997 a 1998 foram influenciados pelo evento El Niño com intensidade forte e, pode ter influência na caracterização dessa seca (Ocorrência de El Niño, 2010). Nota-se que há uma relação causa-efeito entre as anomalias de TSM e os eventos de secas na cidade de Campina Grande.

Tabela 5. Períodos, duração, intensidade e caracterização de secas obtidas pelo SPI-24.

\begin{tabular}{|c|c|c|c|c|c|}
\hline \multicolumn{2}{|c|}{ Anos } & \multirow{2}{*}{$\begin{array}{l}\text { Duração } \\
\text { (meses) }\end{array}$} & \multirow{2}{*}{$\begin{array}{c}\text { Intensidade } \\
\text { do Pico }\end{array}$} & \multirow{2}{*}{$\begin{array}{c}\text { Intensidade } \\
\text { média }\end{array}$} & \multirow{2}{*}{$\begin{array}{c}\text { Categoria de } \\
\text { seca }\end{array}$} \\
\hline Inicial & Final & & & & \\
\hline 1968* & 1972* & $44 *$ & $-1.26 *$ & $-0.56 *$ & Fraca* \\
\hline 1980 & 1981 & 11 & -1.2 & -0.85 & Fraca \\
\hline $1981^{*}$ & $1985^{*}$ & $48^{*}$ & $-2.08 *$ & $-1.14 *$ & Moderada* \\
\hline 1988 & 1990 & 28 & -1.53 & -0.63 & Fraca \\
\hline 1993* & 1995* & $26 *$ & $-2.15^{*}$ & $-0.79 *$ & Fraca* \\
\hline 1997* & 2000* & $38 *$ & $-3.21 *$ & $-1.74 *$ & Severa* \\
\hline
\end{tabular}

$*$ datas, valores e categoria referenciadas no texto deste artigo.

\section{2 - Análises das chuvas}

A Tabela 6 exibe os resultados para o SPI-03, nela se destaca quatro eventos extremos de chuva, sendo o mais intenso de 1963 a 1964, com 13 meses de duração e categoria severa. Os outros três eventos são de intensidade média categorizada como moderada. Há predominância nesses eventos de anos com interferências do fenômeno La Nina. Os demais eventos de chuva são de categoria fraca.

A comparação da ocorrência dos eventos de chuvas com os anos de La Nina revela uma relação entre os fenômenos, principalmente quando a chuva enquadra-se nas categorias moderada ou severa. Em contrapartida, alguns eventos de chuva fraca ou eventos de seca ocorrem em anos de El Nino ou em anos de La Nina, respectivamente. 
Tabela 6. Períodos, duração, intensidade e caracterização de chuvas obtidas pelo SPI-03.

\begin{tabular}{|c|c|c|c|c|c|}
\hline \multicolumn{2}{|c|}{ Anos } & \multirow{2}{*}{$\begin{array}{l}\text { Duração } \\
\text { (meses) }\end{array}$} & \multirow{2}{*}{$\begin{array}{l}\text { Intensidade } \\
\text { do Pico }\end{array}$} & \multirow{2}{*}{$\begin{array}{l}\text { Intensidade } \\
\text { média }\end{array}$} & \multirow{2}{*}{$\begin{array}{c}\text { Categoria de } \\
\text { chuvas }\end{array}$} \\
\hline Inicial & Final & & & & \\
\hline 1962 & 1963 & 13 & 1.44 & 0.51 & Fraca \\
\hline $1963 *$ & $1964 *$ & $13 *$ & $2.43^{*}$ & $1.5^{*}$ & Severa* \\
\hline 1965 & 1967 & 16 & 1.49 & 0.62 & Fraca \\
\hline 1969 & 1969 & 7 & 2.03 & 0.74 & Fraca \\
\hline 1971 & 1972 & 14 & 1.86 & 0.62 & Fraca \\
\hline 1973 & 1974 & 11 & 1.55 & 0.55 & Fraca \\
\hline 1976 & 1977 & 4 & 1.60 & 0.79 & Fraca \\
\hline 1977 & 1977 & 9 & 1.45 & 0.71 & Fraca \\
\hline 1981 & 1982 & 6 & 1.74 & 0.64 & Fraca \\
\hline $1985 *$ & $1985 *$ & 8* & $2.59 *$ & $1.19 *$ & Moderada* \\
\hline 1985 & 1987 & 17 & 1.99 & 0.67 & Fraca \\
\hline 1989 & 1990 & 11 & 1.45 & 0.5 & Fraca \\
\hline 1991 & 1992 & 16 & 1.77 & 0.49 & Fraca \\
\hline 1994 & 1995 & 12 & 2.04 & 0.71 & Fraca \\
\hline 1999* & $2001^{*}$ & $16 *$ & $3.19 *$ & $1.06 *$ & Moderada* \\
\hline $2004^{*}$ & $2004^{*}$ & $11 *$ & $2.77^{*}$ & $1.02 *$ & Moderada* \\
\hline 2005 & 2005 & 6 & 1.46 & 0.72 & Fraca \\
\hline 2007 & 2007 & 4 & 1.13 & 0.52 & Fraca \\
\hline 2009 & 2009 & 10 & 1.63 & 0.81 & Fraca \\
\hline
\end{tabular}

* datas, valores e categoria referenciadas no texto deste artigo.

A Tabela 7 exibe os resultados para o SPI-06. Nela pode ser visto que houve uma redução acentuada no número de eventos de chuva. Esse fato demonstra que há, na cidade de Campina Grande, um padrão pluviométrico pouco variável em torno da média climatológica. Dos eventos destacados no SPI-03, Tabela 6, apenas três se mantiveram no SPI-06, Tabela 7. Destaca-se o evento de 1984 a 1987, na categoria de chuva fraca (chuva acima da média) com duração de 37 meses, muito superior aos demais eventos.

Tabela 7. Períodos, duração, intensidade e caracterização de chuvas obtidas pelo SPI-06.

\begin{tabular}{|c|c|c|c|c|c|}
\hline \multicolumn{2}{|c|}{ Anos } & \multirow{2}{*}{$\begin{array}{l}\text { Duração } \\
\text { (meses) }\end{array}$} & \multirow{2}{*}{$\begin{array}{c}\text { Intensidade } \\
\text { do Pico }\end{array}$} & \multirow{2}{*}{$\begin{array}{l}\text { Intensidade } \\
\text { média }\end{array}$} & \multirow{2}{*}{$\begin{array}{c}\text { Categoria de } \\
\text { chuvas }\end{array}$} \\
\hline Inicial & Final & & & & \\
\hline 1963* & $1965 *$ & $15 *$ & $2.21 *$ & $1.55^{*}$ & Severa* \\
\hline 1966 & 1967 & 23 & 1.49 & 0.50 & Fraca \\
\hline 1969 & 1969 & 7 & 1.57 & 0.88 & Fraca \\
\hline 1984* & $1987 *$ & $37 *$ & $2.23 *$ & $0.72 *$ & Fraca* \\
\hline 1994 & 1995 & 10 & 1.86 & 1 & Moderada \\
\hline 2000 & 2002 & 32 & 2.89 & 0.74 & Fraca \\
\hline 2004* & $2005 *$ & $15 *$ & $2.53^{*}$ & $1.03 *$ & Moderada* \\
\hline
\end{tabular}

* datas, valores e categoria referenciadas no texto deste artigo. 
O SPI-12 detectou seis ocorrências de períodos anuais de precipitação acumulada acima da média, Tabela 8 , todas na categoria fraca. Os três períodos chuvosos destacados no SPI-06, Tabela 7, se mantiveram também nesta escala de 12 meses. Dentre eles, dois apresentaram as maiores intensidades médias e o terceiro apresentou a maior duração, 41 meses, entre os anos 2004 e 2007. O maior pico, 2,46, foi verificado entre os anos de 1962 e 1965, intervalo com predominância de eventos La Nina.

Tabela 8. Períodos, duração, intensidade e caracterização de chuvas obtidas pelo SPI-12.

\begin{tabular}{cccccc}
\hline \multicolumn{2}{c}{ Anos } & $\begin{array}{c}\text { Duração } \\
\text { Inicial }\end{array}$ & $\begin{array}{c}\text { Intensidade } \\
\text { do Pico }\end{array}$ & $\begin{array}{c}\text { Intensidade } \\
\text { média }\end{array}$ & $\begin{array}{c}\text { Categoria de } \\
\text { chuvas }\end{array}$ \\
\hline $1962 *$ & $1965^{*}$ & $33^{*}$ & $2.46 *$ & $0.83^{*}$ & Fraca* \\
1977 & 1979 & 21 & 0.9 & 0.54 & Fraca \\
$1984^{*}$ & $1987 *$ & $37 *$ & $1.89 *$ & $0.96 *$ & Fraca* \\
1991 & 1993 & 24 & 1.31 & 0.58 & Fraca \\
2000 & 2003 & 35 & 2.28 & 0.71 & Fraca \\
$2004 *$ & $2007 *$ & $47^{*}$ & $1.95^{*}$ & $0.52^{*}$ & Fraca* \\
\hline
\end{tabular}

$*$ datas, valores e categoria referenciadas no texto deste artigo.

A Tabela 9, SPI-24, mostra as ocorrências de quatro longos períodos de chuvas acumuladas acima da média. Em todos esses períodos houve a atuação do fenômeno La Nina. Esse fato confirma a influência do fenômeno na região, potencializando a magnitude da precipitação. Diferentemente do SPI-12, o SPI-24 detectou um evento de chuva moderada no período de 1985 a 1988, com grande intensidade de pico, 2,34, e duração de 38 meses.

Tabela 9. Períodos, duração, intensidade e caracterização de chuvas obtidas pelo SPI-24.

\begin{tabular}{|c|c|c|c|c|c|}
\hline \multicolumn{2}{|c|}{ Anos } & \multirow{2}{*}{$\begin{array}{l}\text { Duração } \\
\text { (meses) }\end{array}$} & \multirow{2}{*}{$\begin{array}{c}\text { Intensidade } \\
\text { do Pico }\end{array}$} & \multirow{2}{*}{$\begin{array}{l}\text { Intensidade } \\
\text { média }\end{array}$} & \multirow{2}{*}{$\begin{array}{c}\text { Categoria de } \\
\text { chuvas }\end{array}$} \\
\hline Inicial & Final & & & & \\
\hline 1963* & 1969* & $62 *$ & $1.66^{*}$ & $0.83 *$ & Fraca* \\
\hline $1985 *$ & 1988* & $38 *$ & $2.34 *$ & $1.14 *$ & Moderada* \\
\hline 2000 & 2003 & 34 & 1.78 & 0.84 & Fraca \\
\hline $2004^{*}$ & $2007 *$ & $43 *$ & $1.62 *$ & $0.58^{*}$ & Fraca* \\
\hline
\end{tabular}

$*$ datas, valores e categoria referenciadas no texto deste artigo.

\section{CONCLUSÃO E SUGESTÕES}

Os SPIs não detectaram, segundo a intensidade média, categorias de secas e chuvas extremas na cidade de Campina Grande. A categoria mais freqüente entre os SPIs foi à de chuva normal. Em termos quantitativos as chuvas anuais na cidade de Campina Grande sempre ocorrem em torno do valor esperado. Essa esperança matemática ocorre com maior freqüência pelo lado negativo da escala em torno do valor médio. Esse é o motivo da característica semiárida da Cidade. Nos últimos 50 anos, apenas três registros de precipitação anual abaixo de $500 \mathrm{~mm}$. Pode-se afirmar que há 
garantia, com $94 \%$ de probabilidade, de que as chuvas anuais na Cidade serão superiores a $500 \mathrm{~mm}$.

Os anos que apresentaram maiores e menores índices de pluviometria foram detectados pelo SPI-12 meses e guardam uma boa relação com os eventos La Niña e El Niño, respectivamente. As secas dos períodos de 1968 a 1971, de 1988 a 1989 e de 1997 a 2000 foram identificadas por todos os SPIs estudados. Da mesma forma, os períodos de 1963 a 1969 e de 2004 a 2005 foram detectados como chuvosos por todos os SPIs. As maiores intensidades médias verificadas para as secas e chuvas foram 1,75 pelo SPI - 12 meses, no período de 1993 a 1994, e de 1,55, pelo SPI06 meses, no período de 1963 a 1965, respectivamente.

\section{AGRADECIMENTOS}

Os autores agradecem ao Conselho Nacional de Desenvolvimento Científico e Tecnológico (CNPq) pelo apoio financeiro concedido para elaboração desta pesquisa e a Universidade Federal de Campina Grande (UFCG) pela cessão dos dados.

\section{REFERÊNCIAS}

.AZEVEDO, P. V.; SILVA, V. P. R. Índice seca para a microrregião do agreste da borborema no estado da Paraíba. Revista Brasileira de Meteorologia, v.9, n.1, p. 66-72, 1994

.BLAIN, G. C; BRUNINI, O. Análise Comparativa dos índices de seca de Palmer, Palmer Adaptado e índice padronizado de precipitação no estado de São Paulo. Revista Brasileira de Meteorologia, v.22, n.1, p.105-111, 2007.

.BRASIL. Ministério da Integração Nacional/SDR. Relatório Final do Grupo de Trabalho Interministerial para Redelimitação do Semi-árido Nordestino e do Polígono das Secas. Brasília, DF, 2005. p.118.

.CERQUEIRA, H. D. V. Modulação da temperatura da superfície do mar do pacífico e atlântico tropical na precipitação no estado da Paraíba. Dissertação (Mestrado em Meteorologia) - Universidade Federal de Campina Grande, Campina Grande, 2010.

.CPTEC. Disponível em: <http://enos.cptec.inpe.br/>. Acesso em: 13/09/2010.

.DOMINGOS, S. I. S. Análise do índice de seca Standardized Precipitation Index (SPI) em Portugal Continental e sua comparação com o Palmer Drought Severity Index (PDSI). Tese de licenciatura (Meteorologia) - Universidade de Lisboa, Lisboa, 2006.

.HUGHES, B.L.; SAUNDERS, M.A. A Drought climatology for Europe. International Journal of Climatology , v.22, p.1571-1592, 2002.

.IBGE. Disponível em: <http://www.ibge.gov.br/cidadesat/painel/>. Acesso em: 01/09/2011.

.MACEDO, M. J. H.; Guedes, R. V. S.; Sousa, F. A. S.; Dantas, F. R. C. Análise do índice padronizado de precipitação para o estado da Paraíba, Brasil. Revista Ambiente \& Água, v.5, n.1, p.204-214, 2010.

.MCKEE, T. B.; DOESKEN, N. J.; KLEIST, J. The relationship of drought frequency and duration of time scales. Eighth Conference on Applied Climatology, American Meteorological Society, Jan 17-22, Anaheim CA, p.179-186, 1993.

.NETO, J. M. M.; BARBOSA, M. P.; ARAÚJO A. E. Efeito dos eventos ENOS e das TSM na variação pluviométrica do semi-árido paraibano. Revista Brasileira de Engenharia Agrícola e Ambiental, v.11, n.1, p.61-66, 2007.

.PRESS RELEASE No. 872. Disponível em: http://www.wmo.int/pages/media centre/press_releases/pr_872_en.html. Acesso em: 03/03/2010.

.PEREIRA, I. M.; ANDRADE, L. A.; BARBOSA, M. R. V.; SAMPAIO, E. V. S. B. Composição florística e análise fitossociológica do componente arbustivo-arbóreo de um remanescente florestal no Agreste Paraibano. Acta Botânica Brasilica, v.16, n.3, p. 357-369, 2002.

.RODRIGUEZ, J. L. Atlas Escolar da Paraíba. 2a Ed., Ed. Grafset, João Pessoa-PB, 2000.

.TONKAZ, T. Spatio-temporal assessment of historical droughts using SPI with GIS in GAP Region, Turkey. Journal of Applied Sciences, v.12, n.6, p. 2565-2571, 2006.

.TSAKIRIS, G; VANGELIS, H. Towards a Drought Watch System based on Spatial SPI. Water Resources Management, v.18, p. 1-12, 2004. 